\title{
Circularly Polarized Light on Graphene with Trigonal Warping
}

\author{
Defne AKAY* \\ Department of Physics, Faculty of Sciences, Ankara University, 06100, Ankara, Turkey
}

(ORCID: 0000-0001-8990-007X)

\begin{abstract}
In this article, we theoretically investigate the electronic band structure of monolayer graphene in the presence of trigonal warping and photo-induced effects. The total Hamiltonian of the system has been written and the optical absorption of circularly polarized light for the high frequency regime have been modelled by the Haldane interaction. The relation between trigonal warp aspects and optical absorption of circularly polarized light has been overviewed through the model. Additionally, theoretically analyzed the versatile electronic properties of trigonal warped-graphene under circularly polarized light. We have concluded that photo-induced effect which induced circularly polarized light leads to the opening of energy gap between valance and conduction bands while raises electron-hole asymmetry in the system.
\end{abstract}

Keywords: Circularly Polarized Light, Graphene, Trigonal Warp.

\section{Üçgensel Eğrilikli Grafende Dairesel Polarize Işıłk}

\section{$\ddot{O} \mathbf{z}$}

Bu makalede tek tabakalı grafenin elektronik bant yapısını üçgensel eğrilik ve foto-kaynaklı etkiler varlığında teorik olarak araştırdık. Sistemin toplam Hamiltoniyeni yazılmış ve yüksek frekans rejimi için dairesel polarize ışığın optik absorpsiyonu Haldane etkileşimi ile modellenmiştir. Üçgensel eğrilik bakış açısı ile dairesel polarize ışığın optik absorpsiyonu arasındaki ilişki model üzerinden genel olarak incelenmiştir. Ayrıca, dairesel polarize ışık altındaki üçgensel eğrilikli grafenin çok yönlü elektronik özelliklerini teorik analiz edildi. Dairesel polarize 1şığı indükleyen foto-kaynaklı etkinin, sistemde elektron boşluk asimetrisi üretirken, valance ve iletken bantlar arasında enerji boşluğunun açılmasına yol açtığı sonucuna varıldı.

Anahtar kelimeler: Dairesel Polarize Işık, Grafen, Üçgensel Eğrilik.

\section{Introduction}

Graphene is a one-atom-thick layer of carbon atoms which is forming a two-dimensional honeycomb lattice. The structure has a variety of unusual electronic properties. For a long time graphene has been considered as a theoretically but later on it became an attractive research area by the experimental fabrication of the structure. Pristine graphene has low energy excitations which are defined by a two dimensional massless Dirac equation [1-4] and its charge carriers have low energy spectrum. The charge carriers have a conical band structure and preserve the valley degeneracy. In this material, iso-energetic band dispersion has circular forms [5] but in the presence of trigonal distorsion, iso-energetic band dispersion is modified. The circular forms tend to be trigonal forms due to the lattice symmetry and changed the topology of low energy bands at the $K$ and $K^{\prime}$ valley points in the Brillouin zone of graphene. This phenomenon is called as a trigonal warping (TW) (or distorsion) effect. This feature cause a noticeable results on low energy properties of the graphene, e.g. modifies nature of the conductivity near the Dirac points of graphene and its transport characteristic which has been investigated experimentally [6] and many aspects of theoretically [7-9]. Trigonal warping, also drastically changes the minimal conductivity value that is predicted to be $\sigma \cong 12 e^{2} / \pi^{2} \hbar$ in the presence

"Sorumlu yazar:dakay@science.ankara.edu.tr

Geliş Tarihi: 17.07.2019, Kabul Tarihi: 05.12.2019 
of bilayer graphene [10]. For an all-inclusive investigation of the transport properties of monolayer graphene and bilayers, see [11,12]. In 1988 Haldane [13] introduced a model which exhibited striking properties on the graphene. The effects of photo-irradiation induced by Haldane interaction is quite interesting $[14,15]$. This interaction corresponds to the integer quantum Hall(IQH) effect [16] because it has two bands separated by two fold given potential [17,18]. The potential represented by the mass term which is related to the parity anomaly substantiation in $(2+1)$ dimensional relativistic field theory. Haldane's model can be implemented by realization a periodic magnetic field but which has no net magnetic flux per unit cell and in this model spins violate of time reversal (TR) symmetry which is equal to the model for spinless electrons. Hence, IQH effect can be generalized to cases without an applied magnetic field by a TR symmetry breaking term in the Hamiltonian and this exemplify the Chern insulators and which give rise to an opposite sign at points of $\mathrm{K}^{\prime}$ and $\mathrm{K}$. To the best of our knowledge there has not been an informant on the circularly polarized light on the trigonal distorted monolayer graphene, yet. In this paper, we have performed analytical study on the Landau level structure of the monolayer graphene to figure out circularly polarized light effect on the trigonal distorted graphene. In particular, we have focused on the analysis with the existence of both effects (the gap opening term Haldane interaction and trigonal warping term) and we have presented how to modify the Landau levels and Fermi lines topology of the graphene.

\section{Material ve Methods}

In a graphene, Fermi surface comprises two points. The point states are labelled by the direction of a wave vector $K$ and a unit cell contains two carbon atoms identified as A and B. Dirac equation for the effective low-energy quasi-particle states in the presence of trigonal warping and photo-induced effect, in the vicinity of the K-point of the Brillouin zone, can be written as,

$H=\left(\begin{array}{cc}\Delta^{\tau} & H_{1}+H_{2} \\ -H_{1}^{\dagger}+H_{2}^{\dagger} & -\Delta^{\tau}\end{array}\right)$

The effective Hamiltonian is given by Eq.(1), where $H_{1}$ represents the electronic part, can be given by

$H_{1}=\gamma\left(k_{x}-i k_{y}\right)$

where $\gamma=\hbar v_{F}$ is the band parameter and $k$ is the wave vector. This Hamiltonian contains two different kind of bands, the $\sigma$ and $\pi$ bands. Mechanical properties of graphene are formed by the $\sigma$ bands while unique electronic properties are formed by the $\pi$ bands which are created by the $\pi$ bonds between the out-of-plane $p_{z}$-orbitals. $\pi$ bands has cone-like dispersion relation are described by the Dirac-type $2 \times$ 2 Hamiltonian in the vicinity of the inequivalent points, i.e., $K^{\prime}$ and $K$ valleys. The spectrum of this Hamiltonian $\varepsilon=\sigma \hbar v_{F} k . H_{2}$ term introducing a trigonal distorsion known as trigonal warping which breaks the isotropy of dispersion relation at low energy and this can be also important since it changes the topology of the energy bands close to the Dirac point.

$H_{2}=\gamma \bar{\beta} \exp (3 i \eta)\left(k_{x}+i k_{y}\right)^{2}$

Here $\bar{\beta}$ is described as $\bar{\beta}=\beta a_{c c} / 4 \hbar$ and here, $a_{c c}$ is the lattice constant of graphene. $\beta$ is a constant of the unity order, which can be ensured by a tight binding model[19], the values of $\beta$ is 1 . For $\beta=0$ there is no trigonal warping. This parameter have been also evaluated in metallic carbon nanotubes[20]. According to theoretical and experimental data, it is clearly known that band dispersion of graphene and carbon nanotubes show similarity [21]. $\eta$ represents the chiral angle that specifies the material. If $\eta=0$ is selected, graphene can be described as a zigzag graphene, while $\eta=\pi / 6$ is selected, graphene can be described as an armchair graphene. Photo-irradiation effect is included circularly polarized light and modeled Haldane potential, we have to take into account the edge states. But in this situation effective-energy term characterizing the impacts of circularly polarized light, which renormalized the mass of the Dirac fermions can be described as $\Delta=\left(\hbar v_{F}\right)^{2} A^{2} / \hbar \Omega$. Where $A$ is the defining the intensity of light with frequency $\Omega=3500 \mathrm{THz}$ [22]. 
here, $\tau$ is the valley index which acts differently on the inequivalent $\mathrm{K}$ and $\mathrm{K}^{\prime}$ points. Additionally, it also depends on the application direction of the light. If circular polarization applied on structure, the band gap is reduced in $\mathrm{K}^{\prime}$ valley and increased in the $\mathrm{K}$ valley while for other-side application of circular polarization, the result can be reversed. The normalized eigenvector of the system in $\mathrm{K}$ valley can obtained as,

$\Psi^{K}=\frac{1}{\sqrt{2 E^{K}\left(E^{K}+\Delta\right)}}\left(\begin{array}{c}\Delta+E_{v}^{K} \\ \gamma k_{+}+\gamma \bar{\beta} \exp (-3 i \eta) k_{-}^{2}\end{array}\right)$

The energy bands defined by the application of $\mathcal{H} \Psi=E \Psi$ eigenvalue equation and analytically calculated eigenvalue result can be obtained as,

$E^{K}(\boldsymbol{k})=\sqrt{\Delta^{2}+\gamma^{2} k^{2}+\gamma^{2} \bar{\beta}^{2} k^{4}+2 \gamma^{2} \bar{\beta} k^{3} \operatorname{Cos} 3[\eta+\theta]}$

$E_{v}^{K}(\boldsymbol{k})=v E^{K}(\boldsymbol{k})$

here $v$ is the band index, Eq.(6) is the band dispersion, i.e., energy eigenvalues of the system. The band dispersion of $\mathrm{K}^{\prime}$ can be easily obtained by just rotating $\theta$ by amount of $\pi / 3$ and the $k \cdot p$ equation for $\mathrm{K}^{\prime}$ point can be obtained by the replacement of $H_{1}$ with $-H_{1}^{\dagger}$ and $H_{2}$ with $-H_{2}^{\dagger}$. Haldane term $\Delta^{\tau}$ have must replace with $-\Delta^{\tau}$. So this means that the consideration only one of the Dirac valley is enough to evaluate the energy band dispersion relation of graphene in the presence of both Haldane interaction and trigonal warp effects. Circularly polarized light have been exemplified for germanene and its band structure have been analyzed in the high frequency regime [23]. Ezawa [24], has also investigated the effects of Haldane potential to show the photo-induced effects result of topological phase(TP) transition from a topological insulator to another topological insulator by irradiating circular polarized light in silicene. So it can easily understand that photo-induced effect changes the topological class of topological insulators. Additionally, Liu et al. [25] have also gave a brief overview of the quantum Hallinsulator property on silicene. Drummond et al. [26] have examined the photoinduced topological phase transition and showed that the effect has been controlled by applying the electric field perpendicular to the material sheet. Considering the values and analyzed of these studies, there is not yet an exact clarification for all nano-based structures and devices. Taking into account of this investigations, we have adopted circularly polarized light on the band structure of graphene within the k.p model in the high frequency regime. For this reason, we would like to contribute to the literature with possible explanations. Fermi lines topology which are close to the Dirac points with trigonal warping, see Fig. 1(a) and 1(b), $K$ and $K^{\prime}$ valley of the graphene are related to each other by TR symmetry. According to this figure, trigonal distortion strongly effects the circular band structure of graphene and each the Fermi line about $K$ and $K^{\prime}$ is broken. In Figure 1(a) and 1(b), we have examined the difference of these energies with $K$ and $K^{\prime}$ valley of the graphene by the use of the expression of $\Delta E(\boldsymbol{k})=E^{K}(\boldsymbol{k})-E^{K^{\prime}}(\boldsymbol{k})$ and $\Delta E(\boldsymbol{k})=E^{K^{\prime}}(\boldsymbol{k})-E^{K}(\boldsymbol{k})$, respectively. In the figures Haldane interaction strength is accepted as a zero to see the only effect of TW. Fermi lines analyzed $k_{x}=k_{y}$ condition so this gives the symmetric effect on the Dirac points. One switches on the circularly polarized light the structure, see Fig.(1a) and Fig.(1b), the band gap reduces in one of the graphene valley, while it increases in the other, i.e., the two valley reflecting opposite signs of the effective mass term. In short, it can be easily seen that $K^{\prime}$ and $K$ valley of the graphene affected by an opposite effect. Figures 2 exhibit the topology of the Fermi contour lines that are close to the $K$ valley of the graphene in the presence of combined effects of Haldane type (mass) interaction and trigonal warping. In these figures, we have compared the Figure 2(a) ( $\Delta=4 \times$ $0.0037 \mathrm{meV})$, Figure 2(b) ( $\Delta=0.0037 \mathrm{meV})$ in the $K$ valley of the graphene. It is seen that the strength of the photo-irradiation highly effects the energy level of Fermi lines of the graphene. For a better understanding of the energy level changes with photo-irradiation, we also provided plots in Fig.3 in the $K^{\prime}$ valley of the graphene. From these figures, it is easy to see how the level spacing increases by increasing the strength of Haldane mass term as in the Figures 2. As presented in Figures 2 and Figures 
3 , the low energies of the trigonal band structure show an anisotropic behavior on the two valley. When the interaction amplitude of polarized light expands from $\Delta=0.0037 \mathrm{meV}$ to $\Delta=4 \times 0.0037 \mathrm{meV}$ in Fig. 2(a) for the $K$ valley ( $\Delta=0.0037 \mathrm{meV}$ to $\Delta=4 \times 0.0037 \mathrm{meV}$ in Fig.2(a) for the $K^{\prime}$ valley) showing that the contour splits at low energy. We can seen from that photo-induces effect treats like a homogeneous magnetic on the structure and the band gap shows the nontrivial edge states, which demonstrates a TP transition.
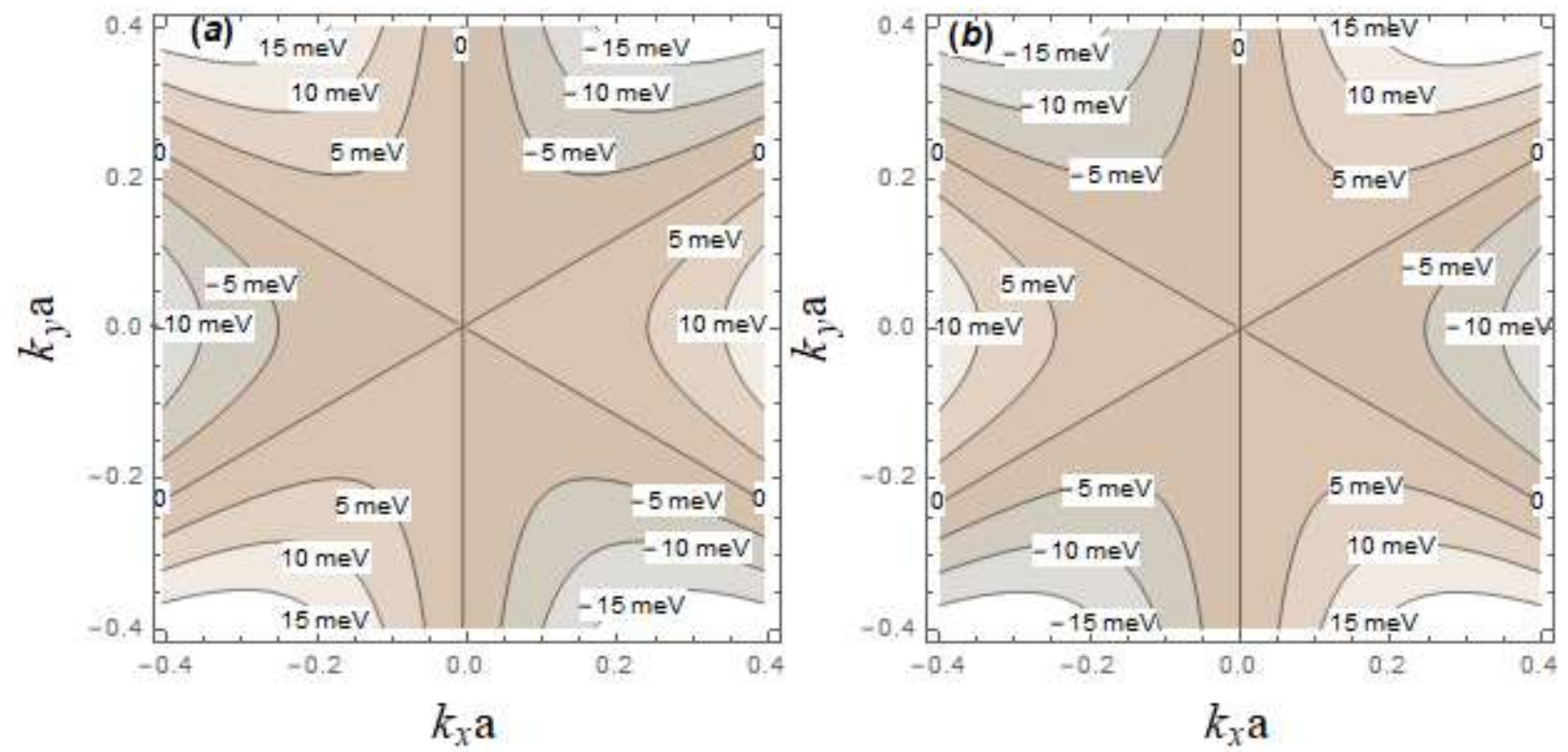

Figure 1. (Color online) Fermi lines topology, which are near to the Dirac points with TW (a) $K$ and (b) and $K^{\prime}$ valley of the graphene are pertinent to each other by time reversal symmetry. Numbers of the contours indicate the energy in unit
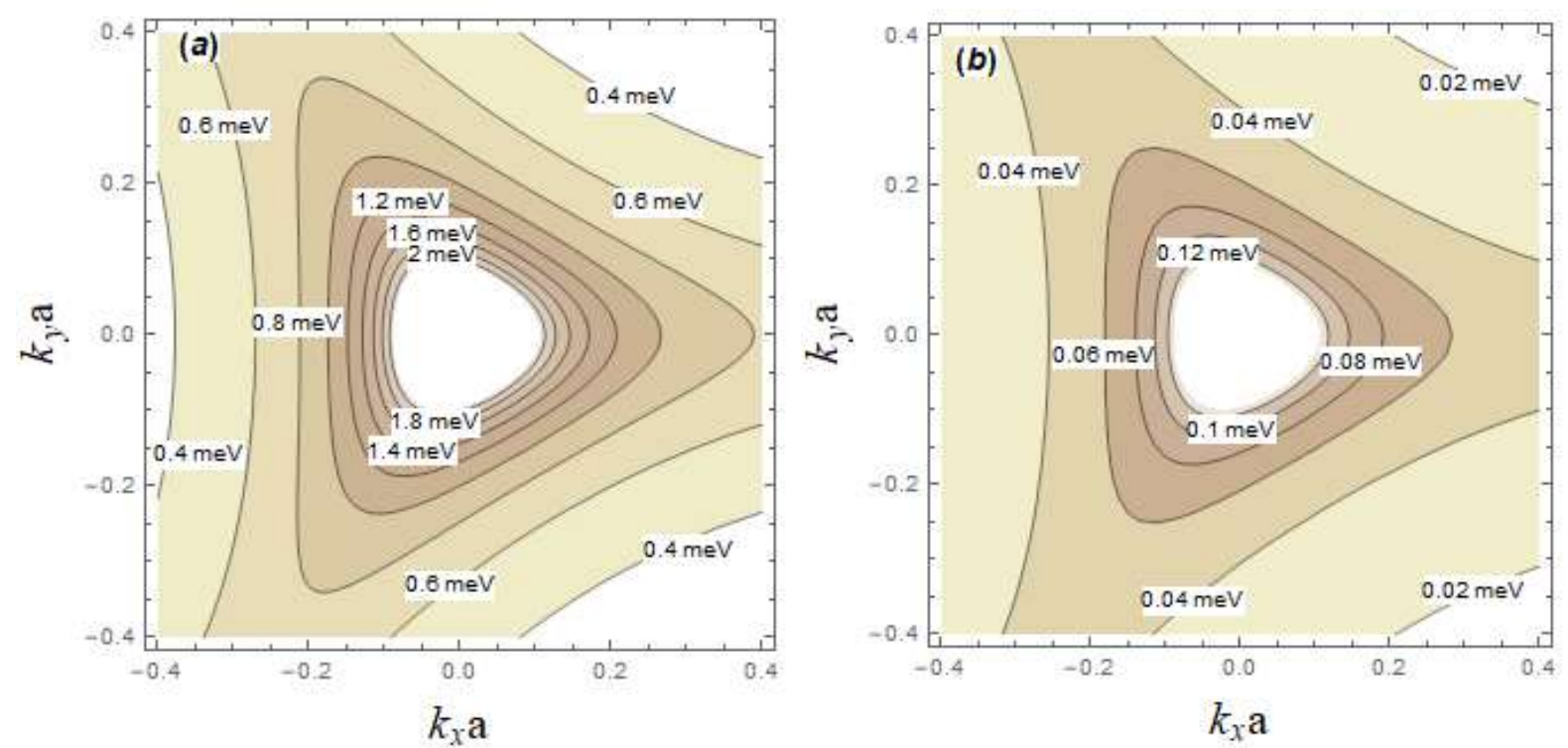

Figure 2. (Color online) Fermi lines topology which are close to the $\mathrm{K}$ valley of the graphene for different values of Haldane mass term (a) $\Delta=4 \times 0.0037 \mathrm{meV}$, (b) $\Delta=0.0037 \mathrm{meV}$. (Numbers of the contours indicate the energy difference in unit) 


\section{Summary and Conclusions}

The outcomes of the present study allow us to draw a conclusion that the photo-induced effect and trigonal warping on the graphene provides us an effective tool for tuning the topology of the Fermi lines which are near to the Dirac cones. Trigonal warping causes a significant modifications to the single electron dispersion because a trigonal distortion that breaks the $k \rightarrow-k$ symmetry of the Fermi lines, i.e., produces asymmetry of the dispersion at each valley $E(K, k) \neq E(K,-k)$. We have been investigated a photo-induced TP transition from a topological insulator to another because the band structure is altered by the dressing of circular photon, where the topological property is modified. Based on the presented study, the application of controlling the topology of the Fermi lines can be also applicable for graphene in the presence of a homogeneous magnetic field. Thus, the energy splitting has been compared with photo-induced effects and magnetic fields because we see that the contour splitting in the presence of Haldane mass. Additionally, the effects of trigonal distortion on the multilayer graphene [27-29] more effective than the monolayer graphene. The simultaneous effects of these two potentials can be analyzed on the multilayer graphene by taking into account of interlayer relation. A large and growing body of literature can be extended with spin-orbit interactions which corresponding to the homogeneous electric field and also be exert together with intrinsic spin-orbit interaction.

\section{Author's Contributions}

All contribution (resources, data collection and/or processing, analysis, literature search and writing manuscript) belongs to the author.

\section{Statement of Conflicts of Interest}

No potential conflict of interest was reported by the author.

\section{Statement of Research and Publication Ethics}

The author declares that this study complies with Research and Publication Ethics.

\section{References}

[1] Wallace P.R. 1947. The Band Theory of Graphite. Phys. Rev., 71: 622.

[2] Semenof G.W. 1984. Condensed-Matter Simulation of a Three-Dimensional Anomaly. Phys. Rev. Lett., 53: 2449.

[3] Geim A.K., Novoselov K.S. 2007. The rise of graphene. Nat. Mater., 6: 183.

[4] Castro Neto A.H., Guinea F., Peres N.M.R., Novoselov K.S., Geim A.K. 2009. The electronic properties of graphene. Rev. Mod. Phys., 81: 109.

[5] Abergel D., Berashevich J., Ziegler Z., Chakraborty T. 2010. Properties of graphene: a theoretical perspective. Adv. Phys., 59 (4): 261-482.

[6] Zhang Y., Tan Y.-W., Stormer H.L., Kim P. 2005. Experimental observation of the quantum Hall effect and Berry's phase in graphene. Nature, 438: 201.

[7] Cserti J., Csord'as A., D'avid G. 2007. Role of the Trigonal Warping on the Minimal Conductivity of Bilayer Graphene. Phys. Rev. Lett., 99: 066802.

[8] Moghaddam A.G., Zareyan M. 2009. Anisotropic minimal conductivity of graphene bilayers. Phys. Rev. B, 79: 073401.

[9] Koshino M., Ando T. 2006. Transport in bilayer graphene: Calculations within a self-consistent Born approximation. Phys. Rev. B, 73: 245403.

[10] Cserti J. 2007. Minimal longitudinal dc conductivity of perfect bilayer graphene. Phys. Rev. B, 75: 033405.

[11] Peres N.M.R. 2010. Colloquium: The transport properties of graphene: An introduction. Rev. Mod. Phys., 82: 2673.

[12] Das Sarma S., Adam S., Hwang E.H., Rossi E. 2011. Electronic transport in two-dimensional graphene. Rev. Mod. Phys., 83: 407. 
[13] Haldane F.D.M. 1988. Model for a Quantum Hall Effect without Landau Levels: CondensedMatter Realization of the "Parity Anomaly". Phys. Rev. Lett., 61: 2015.

[14] Ryu S., Mudry C., Hou C-Y, Chamon C. 2009. Masses in graphenelike two-dimensional electronic systems: Topological defects in order parameters and their fractional exchange statistics. Phys. Rev. B, 105: 205319.

[15] Ezawa M. 2015. Photo-Induced Topological Superconductor in Silicene, Germanene, and Stanene. J. Supercond. Nov. Magn., 28: 1249.

[16] Novoselov K.S., Jiang Z., Zhang Y., Morozov S.V., Stormer H.L., Zeitler U., Maan J.C., Boebinger G.S., Kim P., Geim A.K. 2007. Room-temperature quantum Hall effect in graphene. Science, 315: 1379.

[17] Ezawa M. 2013. Single Dirac-cone state and quantum Hall effects in a honeycomb structure. EPLA., 104: 27006.

[18] Kitagawa T., Oka T., Brataas A., Fu L.,. Demler E. 2011. Transport properties of nonequilibrium systems under the application of light: Photoinduced quantum Hall insulators without Landau levels. Phys. Rev. B, 84: 235108.

[19] Ajiki H., Ando T. 1996. Energy Bands of Carbon Nanotubes in Magnetic Fields. J. Phys. Soc. Jpn., 65: 1255.

[20] Akimoto K., Ando T. 2004. Effects of Trigonal Warping on Perfect Channel in Metallic Carbon Nanotubes. J. Phys. Soc. Jpn., 73: 2194.

[21] Fathi D. 2011. Review of Electronic Band Structure of Graphene and Carbon Nanotubes Using Tight Binding. Hindawi Publishing Corporation Journal of Nanotechnology, Article ID 471241, $6 \mathrm{p}$.

[22] Zhou X., Xu Y., Jin G. 2015. Anomalous thermomagnetic effects in an epitaxial and irradiated graphene monolayer. Phys. Rev. B, 92: 235436.

[23] Tahir M., Zhang Q.Y., Schwingenschlögl U. 2016. Floquet edge states in germanene nanoribbonsi. Sci Rep. 6: 31821.

[24] Ezawa M. 2013. Photoinduced topological phase transition and a single Dirac-cone state in silicene. Phys Rev Lett., 110 (2): 026603.

[25] Liu C.-C., Jiang H., Yao Y. 2011. Low-energy effective Hamiltonian involving spin-orbit coupling in silicene and two-dimensional germanium and tin. Phys. Rev. B, 84: 195430.

[26] Drummond N.D., Zólyomi V., Fal'ko V.I. 2012. Electrically tunable band gap in silicene. Phys. Rev. B, 85: 075423.

[27] McCann E., Fal'ko V.I. 2006. Landau-Level Degeneracy and Quantum Hall Effect in a Graphite Bilayer. Phys. Rev. Lett., 96: 086805.

[28] McCann E., Koshino M. 2009. Trigonal warping and Berry's phase N $\pi$ in ABC-stacked multilayer graphene. Phys. Rev. B, 80: 165409.

[29] McCann E., Smirnov D., Bao W., Jing L., Velasco J., Lee Y., Liu G., Tran D., Standley B., Aykol M., Cronin S. B., Koshino M., Bockrath M., Lau C. N. 2011. Stacking-dependent band gap and quantum transport in trilayer graphene. Nat. Phys., 7: 948. 